\title{
Effectiveness of epicardial atrial pacing using a bipolar steroid-eluting endocardial lead with active fixation in an experimental model ${ }^{1}$
}

\author{
Sávia Christina Pereira Bueno', Wagner Tetsuji Tamaki ${ }^{\mathrm{II}}$, Marcelo Fiorelli Alexandrino da Silva ${ }^{\mathrm{III}}$, Cristiane Maciel Zambolim ${ }^{\mathrm{IV}}$, \\ Kátia Regina da Silvav, Martino Martinelli Filhovi, Roberto Costa ${ }^{\text {VII }}$ \\ IFellow PhD degree, Postgraduate Program in Thoracic and Cardiovascular Surgery, Division of Cardiothoracic Surgery, Heart Institute (InCor), \\ Clinics Hospital, Medical School, University of Sao Paulo (USP), Brazil. Study execution, technical procedures, acquisition and analysis of data, \\ manuscript writing. \\ IIPhD, InCor, Clinics Hospital, Medical School, USP, Sao Paulo-SP, Brazil. Design of the study, technical procedures. \\ IIIGraduate student, Medical School, USP, Sao Paulo-SP, Brazil. Technical procedures, acquisition of data.

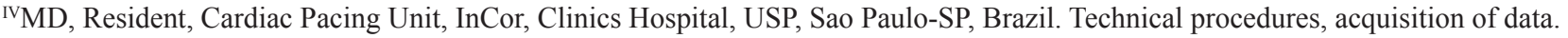 \\ ${ }^{v} \mathrm{PhD}$, Fellow Post-Doctoral degree, InCor, Clinics Hospital, USP, Sao Paulo-SP, Brazil. Design of the study, acquisition and analysis of data, responsible \\ for English language, manuscript writing, critical revision. \\ ${ }^{v i} \mathrm{PhD}$, InCor, Clinics Hospital, USP, Sao Paulo-SP, Brazil. Critical revision. \\ VIIAssociate Professor, Division of Cardiothoracic Surgery, InCor, USP, Sao Paulo-SP, Brazil. Tutor. Conception and design of the study, acquisition \\ and analysis of data, manuscript writing, critical revision.
}

\begin{abstract}
PURPOSE: To assess the effectiveness of bipolar epicardial atrial pacing using an active fixation bipolar endocardial lead implanted on the atrial surface in an experimental model.

METHODS: A total of ten Large White adult pigs underwent pacemaker implantation under general anesthesia. Atrial pacing and sensing parameters were obtained at the procedure, immediate postoperative period and on the 7 th and the 30th postoperative in unipolar and bipolar configurations.

RESULTS: All procedures were successfully performed. There were no perioperative complications and no early deaths. Atrial pacing and sensing parameters for both unipolar and bipolar modes remained stable throughout the study. We observed a progressive increase in atrial thresholds, ranging from $0.49 \pm 0.35$ (at implantation) to $1.86 \pm 1.31$ volts (30th postoperative day), in unipolar mode. Atrial impedance measurements decreased slightly over time, ranging from $486.80 \pm 126.35$ Ohms (at implantation) to $385.0 \pm 80.52$ Ohms (30th postoperative day). Atrial sensing measures remained stable from the immediate postoperative period until the end of the study.

CONCLUSION: The bipolar active fixation endocardial lead implanted epicardially can provide stable conditions of pacing and sensing parameters throughout the postoperative follow-up.

Key words: Pacemaker, Artificial. Surgical Procedures, Operative. Electrodes, Implanted. Transverse Sinuses. Animal Experimentation.
\end{abstract} Swine. 


\section{Introduction}

Unquestionable advantages of transvenous access for atrioventricular pacemaker implantation have made the epicardial access an option used only in exceptional situations, such as in patients with limited venous access as well as in small children ${ }^{1-4}$. This fact has reduced the interest of device manufacturers in developing new leads for implant on the atrial epimyocardial surface. To date, bipolar atrial leads specifically designed for epicardial use are not yet widely available. Therefore some authors have performed atrial epimyocardial implants by using leads routinely applied in endocardial stimulation, adapting them for epimyocardial approach ${ }^{5-7}$.

The implant of an active fixation bipolar endocardial lead on the atrial surface was firstly described in patients with repaired or palliated congenital heart disease by directly penetration of the corkscrew tip to the atrial surface during open chest surgery ${ }^{5}$. More recently, two studies described the use of minimally invasive subxiphoid pericardial access for placement of atrial lead through the transverse sinus under fluoroscopic guidance ${ }^{7,8}$.

The aim of this study was therefore to assess the effectiveness of bipolar epicardial atrial pacing using an active fixation bipolar endocardial lead implanted on the atrial surface in an experimental model.

\section{Methods}

This research protocol was reviewed and approved by the Institutional Review Board of the Clinics Hospital, Medical School, University of Sao Paulo. All animals received humane care in compliance with the Guide for the Care and Use of Laboratory Animals published by the National Institute of Health (NIH, publication 85-23, revised 1996), and we followed the ethics principles in animal research established by the Brazilian College of Animals Research (COBEA) ${ }^{9}$.

\section{Experimental animals}

Between March and November 2011, a total of 10 healthy adult pigs (Large White) with a mean weight of $31.5 \pm$ $2.5 \mathrm{~kg}$ (range $=27$ to $35 \mathrm{~kg}$ ) were submitted to identical surgical procedures at the Laboratory of Experimental Surgery from the Heart Institute (InCor) of the Clinics Hospital of the University of São Paulo Medical School (São Paulo, Brazil). Health status of all animals was assessed before undergoing all experimental procedures. In this protocol the animals were monitored by trained personnel including a specialized veterinarian.

\section{Experimental procedures}

\section{Surgical preparation}

The animals were fasted 12 hours prior to the surgery. Anesthesia was induced with an intramuscular injection of ketamine hydrochloride $(22 \mathrm{mg} / \mathrm{kg})$ and midazolam $(0.3 \mathrm{mg} /$ $\mathrm{kg}$ ) followed by an intravenous administration of etomidate $(0.1$ to $0.4 \mathrm{mg} / \mathrm{kg}$ ). A prophylactic dose of cefazolin (1 gram) was administered immediately before the procedure ${ }^{10}$.

All animals were positioned supine, intubated orally with an endotracheal tube and mechanically ventilated. Artificial respiration was obtained with a volume-control ventilator (Takaoka, model 674). Anesthesia was maintained with intermittent administration of fentanyl $(0.04 \mathrm{mg} / \mathrm{kg}$, IV) and etomidate $(0.2-0.3 \mathrm{mg} / \mathrm{kg}$, IV) in order to achieve a surgical depth of anesthesia. Isoflurane gas $(2 \%)$ was used continuously throughout the procedure and pancuronium $(0.04$ to $0.1 \mathrm{mg} / \mathrm{kg}$ IV) was administered intravenously as necessary ${ }^{10}$.

Central venous catheter for continuous pressure documentation and drug administration, as well as catheterization of the femoral artery for continuous blood pressure monitoring, was obtained in all animals. Conventional surface electrocardiogram (ECG) was continuously monitored throughout the operative procedure.

\section{Surgical procedure}

All operations were performed under general anesthesia and by the same surgical team. After standard skin preparation, a 5-7 cm left anterolateral thoracotomy was made in the fourth left intercostal space. After opening the chest, a transverse incision of approximately $2-3 \mathrm{~cm}$ was made in the pericardial sac, preserving the phrenic nerve. Parietal pericardium was repaired with cotton 2-0 stitches to expose the ventricle and the left atrium.

A bipolar steroid-eluting epicardial pacing lead (Medtronic CapSure Epi 4968 - 35cm) was implanted on the left ventricular free wall with Prolene 5-0 sutures. Pulmonary artery and left atrium were kept away by the use of flexible spacers which allowed the visualization of the medial wall of the right atrium through the transverse sinus. Through the same incision and by using direct visualization, a bipolar steroid-eluting endocardial pacing lead with active fixation (Medtronic CapSureFix Novus $4076-52 \mathrm{~cm}$ ), was directed through the transverse sinus to reach 
the roof of the right atrium, where the electrode was implanted on the atrial epicardium surface, between the superior vena cava and the ascending aorta.

Measurements of atrial and ventricular stimulation threshold, impedance and spontaneous electrical potentials were obtained for both unipolar and bipolar configurations. Pacing thresholds were measured at $0.4 \mathrm{~ms}$ of pulse width. Once satisfactory lead parameters of pacing and sensing were achieved, the pulse generator (Medtronic Adapta ADDR03) was connected to atrial and ventricular leads and the device was placed within a pocket located in the same incision, between the left pectoral muscle and the rib cage. Pericardial or pleural drainage tubes were not used. Fluoroscopic images using $30^{\circ}$ left and right anterior oblique projections as well as anteroposterior view were obtained to confirm adequate leads positioning (Figure 1).

At the end of the procedure, pacemaker was programmed by Medtronic Carelink Model 2090 Programmer. Basically, the following parameters were programmed: DDD mode; lower rate $=60$ pulses per minute $(\mathrm{ppm})$; upper rate limit $=120 \mathrm{ppm}$; atrioventricular interval after a sensed atrial event $=120 \mathrm{~ms}$; atrioventricular interval after a paced atrial event $=180 \mathrm{~ms}$; sensor for rate-adaptive pacing: off; atrial sensing $=0.5 \mathrm{mV}$ in bipolar mode; ventricular sensing $=2.0 \mathrm{mV}$ in bipolar mode.

The postoperative analgesia was obtained with dipyrone $(20 \mathrm{mg} / \mathrm{kg}, \mathrm{IV})$ at the end of the procedure. In case of refractory pain, a supplementary dose of dipyrone $(20 \mathrm{mg} / \mathrm{kg})$ or antiinflammatory drug (diclofenac sodium 20 to $50 \mathrm{mg} / \mathrm{kg}$ ) was administered intramuscularly. All animals were observed three times daily for the first 3 days and remained isolated until the 7 th postoperative day.

Fluoroscopic images showing the atrial lead implanted through the transverse sinus at the roof of the atrial surface (solid arrow) and ventricular lead implanted on the left ventricular free wall (dashed arrow).

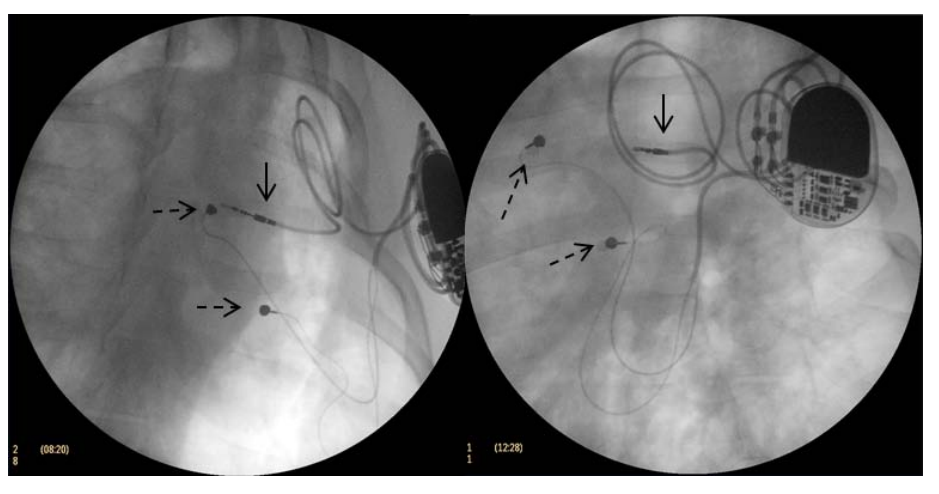

FIGURE 1 - Epimyocardial pacemaker implantation through subxiphoid approach.

\section{$\underline{\text { Postoperative follow-up }}$}

From the 7 th to the 30 th postoperative day after the initial procedure, the animals were housed in a specialized veterinary animal colony.

Pacing and sensing parameters were obtained at the immediate postoperative period and on the 7 th and the 30th postoperative day for both atrial and ventricular leads in unipolar and bipolar configurations: pacing threshold (V) at $0.4 \mathrm{~ms}$ of pulse width, impedance $(\mathrm{Ohm}), \mathrm{P}$ amplitude $(\mathrm{mV})$ and $\mathrm{R}$ amplitude $(\mathrm{mV})$.

\section{Studied variables and statistical analysis}

During each moment of electronic pacemaker evaluations (intraoperative, immediate postoperative, 7 th and 30th postoperative day), for comparison of the atrial lead safety and efficacy, each animal was used as its own control. To characterize the pacing and sensing performance during the first month postimplantation, one-way analysis of variance (ANOVA) for repeated measurements was used for all animals.

Statistical analysis was performed by using Statistical Package for Social Science for Windows (SPSS) and Statistical Analysis System (SAS). Statistical significance was achieved at $\mathrm{p}<0.05$.

\section{Results}

All procedures were successfully performed. There were no perioperative complications and no early deaths. The mean operating time was $177.5 \pm 33.9$ minutes (range $=135$ a 235 minutes). Lead placement on the roof of right atrium through the transverse sinus was possible in all animals. Bleeding or injuries of cardiac and noncardiac structures were not documented.

During the post-implantation week, three minor complications were detected: one animal suffered a superficial wound infection which was treated with antibiotic (cefalotin IM) and local dressings; one animal had dyspnea from the 2nd to 6th postoperative day that ended spontaneously; and finally, one animal had diarrhea, which required hydration and administration of antibiotic (cefalotin IM).

Pacing and sensing parameters for atrial leads in both unipolar and bipolar modes remained stable throughout the study (Table 1). Only in one animal the last pacemaker evaluation was not performed due to cardiac arrest during the sedation administered for its purpose. 
Bueno SCP et al.

TABLE 1 - Atrial pacing thresholds at pacemaker implantation and during the follow-up evaluations.

\begin{tabular}{|c|c|c|c|c|c|c|c|}
\hline \multirow[t]{2}{*}{ Animal } & \multirow[t]{2}{*}{ Phase } & \multicolumn{2}{|c|}{ Capture threshold (V) at $0.4 \mathrm{~ms}$} & \multicolumn{2}{|c|}{ Impedance (Ohm) } & \multicolumn{2}{|c|}{$P$ amplitude (mV) } \\
\hline & & Unipolar & Bipolar & Unipolar & Bipolar & Unipolar & Bipolar \\
\hline \multirow[t]{4}{*}{1} & Intraoperative & 1.33 & 1.50 & 740 & 680 & 4.0 & 3.0 \\
\hline & Immediate PO & 1.00 & 1.25 & 680 & 700 & 2.0 & 2.0 \\
\hline & 7th PO & 2.70 & 3.00 & 323 & 438 & 2.8 & 2.8 \\
\hline & 30th PO & 2.25 & 3.00 & 399 & 528 & 1.4 & 1.4 \\
\hline \multirow[t]{4}{*}{2} & Intraoperative & 0.30 & 0.70 & 393 & 810 & 4.0 & 2.8 \\
\hline & Immediate PO & 0.25 & 0.50 & 340 & 620 & 4.0 & 4.0 \\
\hline & 7th PO & 0.25 & 0.50 & 320 & 431 & 4.0 & 4.0 \\
\hline & 30th PO & 0.75 & 1.00 & 344 & 510 & 5.6 & 5.6 \\
\hline \multirow[t]{4}{*}{3} & Intraoperative & 0.25 & 0.70 & 461 & 650 & 5.6 & 6.0 \\
\hline & Immediate PO & 0.50 & 0.75 & 350 & 500 & 5.6 & 5.6 \\
\hline & 7th $\mathrm{PO}$ & 1.50 & 1.50 & 322 & 420 & 4.0 & 4.0 \\
\hline & 30th PO & 3.50 & 3.75 & 326 & 419 & 5.6 & 5.6 \\
\hline \multirow[t]{4}{*}{4} & Intraoperative & 0.40 & 0.60 & 570 & 650 & 2.9 & 2.9 \\
\hline & Immediate PO & 0.75 & 1.00 & 371 & 471 & 2.0 & 4.0 \\
\hline & 7th PO & 0.50 & 0.75 & 303 & 376 & 2.0 & 2.8 \\
\hline & 30th PO & 2.75 & 3.25 & 296 & 471 & 2.0 & 1.4 \\
\hline \multirow[t]{4}{*}{5} & Intraoperative & 0.10 & 0.63 & 356 & 513 & 5.4 & 4.7 \\
\hline & Immediate PO & 0.25 & 0.50 & 389 & 450 & 2.0 & 4.0 \\
\hline & 7th PO & 0.75 & 1.00 & 274 & 393 & 2.0 & 4.0 \\
\hline & 30th PO & 1.00 & 1.25 & 281 & 397 & 2.0 & 2.0 \\
\hline \multirow[t]{4}{*}{6} & Intraoperative & 0.75 & 0.90 & 318 & 493 & 4.0 & 3.3 \\
\hline & Immediate PO & 1.50 & 1.75 & 265 & 339 & 1.4 & 2.0 \\
\hline & 7th $\mathrm{PO}$ & 3.00 & 4.00 & 237 & 343 & 1.4 & 4.0 \\
\hline & 30th PO & 4.00 & 5.00 & 400 & 507 & 0.7 & 5.6 \\
\hline \multirow[t]{4}{*}{7} & Intraoperative & 0.60 & 0.60 & 530 & 770 & 4.5 & 5.0 \\
\hline & Immediate PO & 0.25 & 0.25 & 423 & 546 & 2.0 & 2.0 \\
\hline & 7th PO & 0.50 & 0.50 & 358 & 397 & 2.0 & 2.8 \\
\hline & 30th PO & 0.50 & 1.00 & 465 & 623 & 1.4 & 2.8 \\
\hline \multirow[t]{4}{*}{8} & Intraoperative & 0.60 & 0.67 & 460 & 540 & 7.4 & 9.0 \\
\hline & Immediate PO & 0.25 & 0.25 & 381 & 486 & 5.6 & 5.6 \\
\hline & 7th PO & 0.50 & 0.75 & 330 & 454 & 5.6 & 5.6 \\
\hline & 30th PO & 0.75 & 1.75 & 501 & 470 & 5.6 & 4.0 \\
\hline \multirow[t]{4}{*}{9} & Intraoperative & 0.30 & 0.62 & 440 & 640 & 8.0 & 5.1 \\
\hline & Immediate PO & 0.25 & 0.50 & 1121 & 1357 & 5.6 & 0.7 \\
\hline & 7th PO & 1.00 & 1.50 & 409 & 635 & 5.6 & 0.7 \\
\hline & 30th PO & 1.25 & 2.00 & 342 & 568 & 5.6 & 5.6 \\
\hline \multirow[t]{4}{*}{10} & Intraoperative & 0.30 & 0.46 & 600 & 700 & 4.0 & 4.0 \\
\hline & Immediate $\mathrm{PO}$ & 0.25 & 0.25 & 389 & 574 & 2.0 & 4.0 \\
\hline & 7th PO & 0.25 & 0.50 & 410 & 823 & 2.8 & 5.6 \\
\hline & 30th PO & - & - & 496 & 741 & - & 2.0 \\
\hline
\end{tabular}

$\mathrm{PO}=$ postoperative 


\section{Atrial capture thresholds}

Mean pacing thresholds showed similar patterns for both unipolar and bipolar lead configurations. There were no statistically significant differences between capture thresholds measured at implantation and at immediate postoperative. However, there was a progressive increase in the mean voltage thresholds when comparing the measurements performed at immediate postoperative and on the 7 th and 30th postoperative day.

Atrial pacing threshold in the unipolar configuration ranged from $0.49 \pm 0.35$ volts (at implantation) to $1.86 \pm 1.31$ volts (30th postoperative day). In bipolar configuration, the atrial pacing threshold ranged from $0.74 \pm 0.29$ volts to $2.44 \pm 1.39$ volts, at implantation and on the 30th postoperative day, respectively.

There was no significant difference between unipolar and bipolar measures performed throughout the study protocol. As shown in Figure 2, the measures of unipolar and bipolar threshold were parallel $(\mathrm{p}=0.817)$ and coincident $(\mathrm{p}=0.343)$.

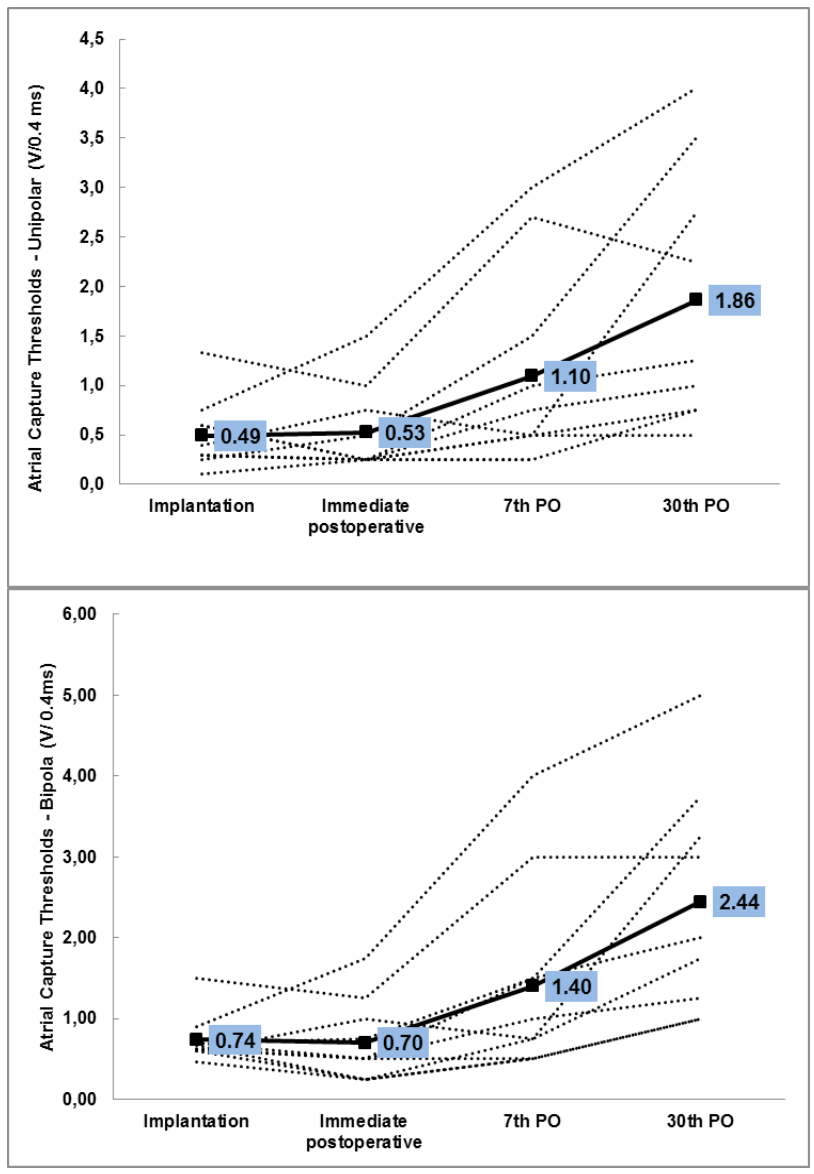

FIGURE 2 - Atrial capture thresholds in unipolar and bipolar lead configurations.

\section{Atrial impedance}

Mean pacing impedance showed similar patterns for both unipolar and bipolar lead configurations. There were no statistically significant changes between pacing impedance measured at implantation and at immediate postoperative. However, there was a progressive decrease of this parameter when comparing the measurements performed at immediate postoperative with the 7 th postoperative day and a slight increase between the 7th and 30th postoperative day.

Atrial pacing impedance in the unipolar configuration ranged from $486.80 \pm 126.35 \mathrm{Ohms}$ (at implantation) to $385.0 \pm$ 80.52 Ohms (30th postoperative day). In bipolar configuration, atrial pacing threshold varied from $644.60 \pm 104.74 \mathrm{Ohms}$ to 523.40 $\pm 101.22 \mathrm{Ohms}$, at implantation and on the 30th postoperative day, respectively.

The comparison between unipolar and bipolar measures showed parallel pattern over time $(p=0.994)$. However, the measures of bipolar impedance were significantly higher than the unipolar atrial impedance $(\mathrm{p}=0.008)$ (Figure 3$)$.

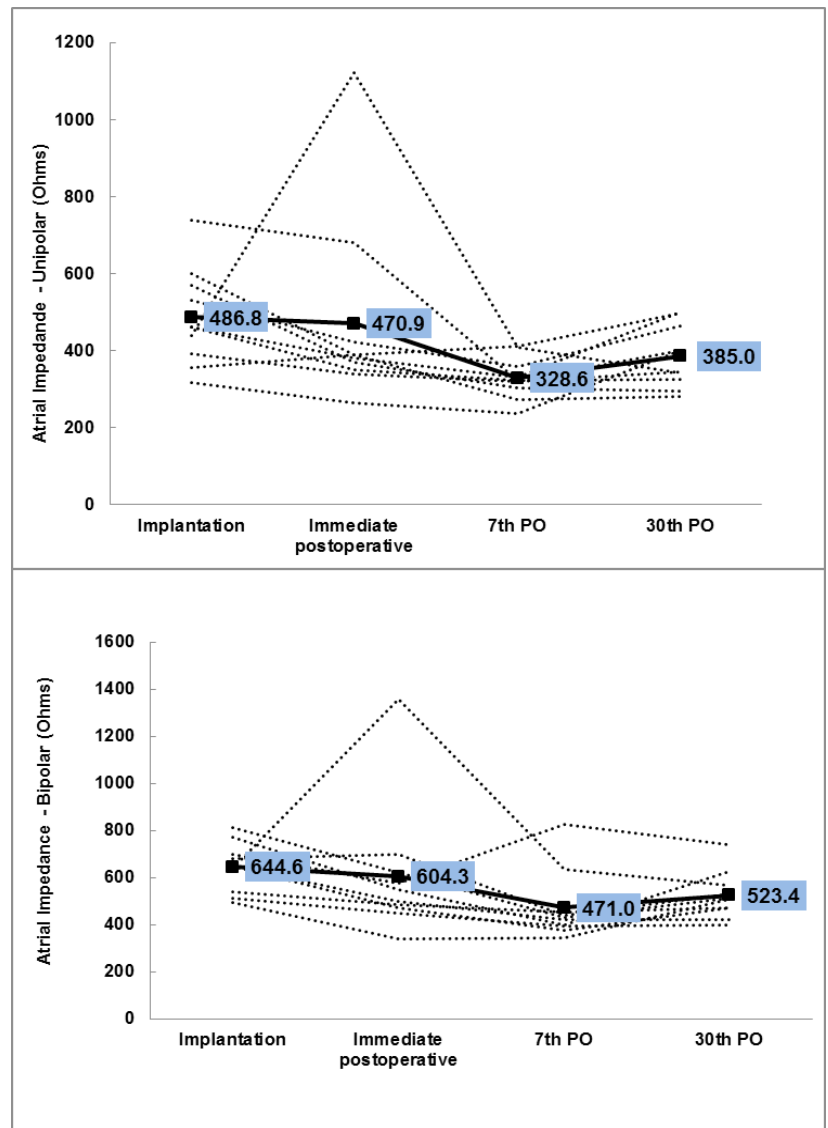

FIGURE 3 - Atrial impedance in unipolar and bipolar lead configurations. 


\section{Atrial sensing}

Mean atrial sensing showed similar patterns for both unipolar and bipolar lead configurations. There was a decrease of this parameter when comparing the measurements performed at implantation and immediate postoperative. Atrial sensing measures remained stable from the immediate postoperative period until the end of the study.

Atrial sensing in the unipolar configuration ranged from $4.97 \pm 1.69 \mathrm{mV}$ (at implantation) to $3.32 \pm 2.19 \mathrm{mV}$ (30th postoperative day). In bipolar configuration, atrial sensing ranged from $4.59 \pm 1.90 \mathrm{mV}$ to $3.60 \pm 1.87 \mathrm{mV}$, at implantation and on the 30 th postoperative day, respectively.

There was no significant difference between unipolar and bipolar measures performed throughout the study protocol. As shown in Figure 4, the measures of unipolar and bipolar atrial sensing were parallel $(\mathrm{p}=0.685)$ and coincident $(\mathrm{p}=0.954)$.

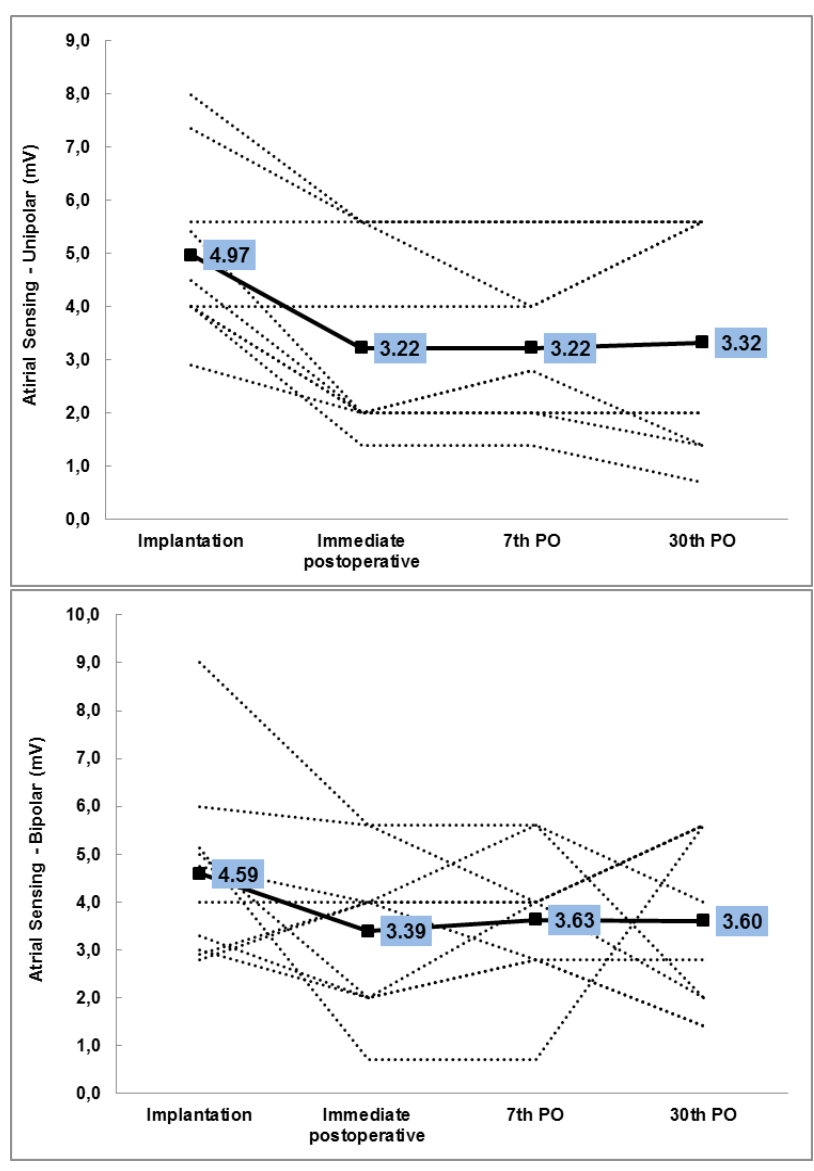

FIGURE 4 - Atrial sensing in unipolar and bipolar lead configurations.

\section{Discussion}

The current animal experimental study, which evaluated the effectiveness of epicardial atrial pacing using a bipolar steroid-eluting endocardial lead with active fixation, showed the feasibility of atrial lead implantation through the transverse sinus by using a minimally invasive subxiphoid approach. This novel implantation technique was successfully performed in all animals without perioperative complications, as well as the absence of lead dislodgement throughout the study, demonstrating the safety and reproducibility of this procedure.

Despite having used a bipolar endocardial lead on the atrial surface, this strategy has provided excellent pacing performance during the study protocol. The effectiveness of this pacemaker was confirmed by observation of pacing and sensing parameters considered stable and compatible with the established measures for both endocardial and epicardial leads implanted through various techniques in the atrium or ventricles ${ }^{11,12}$.

Experience with a novel approach to bipolar epicardial atrial pacing using an active fixation bipolar endocardial lead, as presented in this current study, has not yet been widely reported in the published literature. The performance of an active fixation endocardial lead implanted on the atrial surface was firstly described by Epstein et al. ${ }^{5}$. In this study, 77 active fixation bipolar leads were implanted on the right atrial surface by median sternotomy or anterolateral right thoracotomy in 72 patients with congenital heart disease ranging in age from three months to 38 years (mean $8.9 \pm 8.8$ years). Lead fixation was made by engaging and screwing the lead's helix into the atrial myocardium. Once the corkscrew lead tip was fixed, atrial tissue was imbricated around the proximal electrode with sutures. No perioperative or longterm complications were described and actuarial analysis showed a freedom from failure rate of $98 \%$ at 36 months and $91 \%$ at 48 , 60 , and 72 months.

Costa et $a l .{ }^{6}$ performed the implantation of this type of lead in the atrial roof through the transverse sinus by using a pericardial minimally invasive access in a patient who developed superior vena cava syndrome five years after bi-atrial-ventricular pacemaker implantation. Appropriate lead sensing and pacing parameters were obtained in the intraoperative and remained stable throughout the follow-up period. By using a similar surgical approach, Hsia et al. ${ }^{7}$ performed a minimally invasive placement of a transvenous implantable cardioverter-defibrillator (ICD) lead within the pericardial space in seven children (one to 17 years old) in whom a transvenous access was limited or not feasible. Authors reported no perioperative complications or deaths. The ICD lead 
demonstrated excellent performance during a follow-up period of up to 39 months without inappropriate ICD discharges.

The results of our study demonstrated the stability of atrial pacing threshold measurements in the intraoperative and immediate postoperative period, followed by a progressive increase until the end of the study, reaching an average increase of 2.4 times in the last evaluation, which is compatible with parameters commonly observed in patients and animal studies ${ }^{11}$. According to a canine model, in the first one to three days after lead implantation, there is no evidence of cellular inflammation, and thresholds do not changes significantly ${ }^{13}$. After two to three days, a mixed cellular inflammation begins to appear in the tissues surrounding the electrode, and the stimulation thresholds begins to rise. After three to four post-implantation weeks, the global inflammatory response has essentially resolved and thresholds may subsequently decrease or remain stable, depending on the intensity of the foreign body response at the electrode-tissue interface ${ }^{6,11,13}$. Bognolo et al. ${ }^{14}$ compared the performance of three types of leads (two epicardial and one endocardial) in six dogs. Each dog received one lead on the right atrial appendage, one at the atrial-auricular junction and one on the right atrial wall. The pacing threshold for all three leads increased up to five times when compared to intraoperative measurements with those made at the end of the first week of follow-up. From that point, pacing thresholds remained stable until the end of the first month.

In this study, atrial pacing impedance showed similar patterns with those reported in the literature. Burger et al. ${ }^{15}$ studying the performance of two epicardial steroid-eluting leads showed that the impedances of screw-in leads were significantly lower compared to those of suture-on leads. Slight decrease in impedance values was observed for both types of leads during the early follow-up period, however, impedances remained stable for a period between three and 36 months.

In the same direction, the performance of atrial sensing was similar with previous studies ${ }^{11,12,14}$. The already initially good sensing values $(5 \mathrm{mV})$ showed a decrease in the immediate postoperative but afterwards remained stable during the whole follow-up period.

Our study has two main limitations. First, all procedures were performed by the same surgeon, which may have influenced our surgical results. Second, the lack of a control group did not allow the comparison between the performance of this same lead in endocardial and epicardial implantation sites. However, this comparison can be performed in future studies.

\section{Conclusion}

The bipolar active fixation endocardial lead implanted epicardially can provide acceptable bipolar atrial sensing and pacing. It therefore can be a good approach for atrioventricular pacing in patients for whom endocardial pacing is contraindicated or not feasible.

\section{References}

1. Furman S, Schwedel JB, Robinson G, Hurwitt ES. Use of an intracardiac pacemaker in the control of heart block. Surgery. 1961;49:98-108.

2. Mond HG, Proclemer A. The 11th world survey of cardiac pacing and implantable cardioverter-defibrillators: calendar year 2009--a World Society of Arrhythmia's project. Pacing Clin Electrophysiol. 2011;34(8):1013-27.

3. Lau EW. Upper body venous access for transvenous lead placement review of review of existent techniques. Pacing Clin Electrophysiol. 2007;30:901-9.

4. Belott P, Reynolds DW: Permanent pacemaker and implantable cardioverter-defibrillator implantation. In: Ellenbogen K, Wilkoff B, Kay N, Lau C. Clinical cardiac pacing, defibrillation, and resynchronization therapy. 4ed. Philadelphia: Saunders Elsevier; 2011. p.443-515.

5. Epstein MR, Walsh EP, Saul JP, Triedman JK, Mayer JE, Gamble WJ. Long-term performance of bipolar epicardial atrial pacing using an active fixation bipolar endocardial lead. Pacing Clin Electrophysiol. 1998;21:1098-104.

6. Karpawich PP, Walters H, Hakimi M. Chronic performance of a transvenous steroid pacing lead used as an epi-intramyocardial electrode. Pacing Clin Electrophysiol. 1998;21(7):1486-8.

7. Costa R, Leão MI, Silva KR, Camargo PR, Costa RV. Bi-atrial subxiphoid epicardial pacemaker in superior vena cava syndrome. Arq Bras Cardiol. 2006;87:101-3.

8. Hsia TY, Bradley SM, LaPage MJ, Whelan S, Saul JP, Ringewald $\mathrm{JM}$, Reed JH. Novel minimally invasive, intrapericardial implantable cardioverter defibrillator coil system: a useful approach to arrhythmia therapy in children. Ann Thorac Surg. 2009;87:12349.

9. Colégio Brasileiro de experimentação animal (COBEA). Princípios éticos na experimentação animal. Available in: http://www.univap. br/ipd/docs/principios_eticos_na_experimentacao_animal.pdf.

10. Geovanini GR, Pinna FR, Prado FA, Tamaki WT, Marques E. Padronização da anestesia em suínos para procedimentos cirúrgicos cardiovasculares experimentais. Rev Bras Anestesiol. 2008;58:36370 .

11. Kay NG, Dosdall DJ, Shepard RB. Cardiac electrical stimulation. In: Ellenbogen K, Wilkoff B, Kay N, Lau C. Clinical cardiac pacing, defibrillation, and resynchronization therapy. 4ed. Philadelphia: Saunders Elsevier; 2011. p.3-39.

12. Fortescue EB, Berul CI, Cecchin F, Walsh EP, Triedman JK, Alexander ME. Comparison of modern steroid-eluting epicardial and thin tranvenous pacemaker leads in pediatric and congenital heart disease patients. J Interv Card Electrophysiol. 2005;14:27-36.

13. Anderson JM. Inflammatory response to implants. ASAIO Trans. 1988;34:101-7. 
14. Bognolo D, Stokes K, Wiebush W, Vijayanagar R, Eckstein P, Jeffrey D. Experimental and clinical study of a new permanent myocardial atrial suture less pacing lead. PACE. 1983;6:113-8.

15. Burger H, Kempfert J, van Linden A, Szalay Z, Schoenburg M, Walther T, Ziegelhoeffer T. Endurance and performance of two different concepts for left ventricular stimulation with bipolar epicardial leads in long-term follow-up. Thorac Cardiovasc Surg. 2012;60:70-7.

\section{Correspondence:}

Roberto Costa

Unidade de Estimulação Elétrica e Marcapasso

InCor, Hospital das Clínicas

Faculdade de Medicina-USP

Avenida Doutor Enéas de Carvalho Aguiar, 44

05403-900 São Paulo - SP Brasil

Tel.: (55 11)2661-5284

rcosta@incor.usp.br

Received: March 11, 2013

Review: May 13, 2013

Accepted: June 14, 2013

Conflict of interest: none

Financial sources: National Council of Scientific and Technological Development (CNPq), Scholarship (Process number: 508370/2010-8) and Medtronic of Brazil

${ }^{1}$ Research performed at Cardiac Pacing Unit, Cardiopneumology Department, School of Medicine, Sao Paulo University (USP), Brazil. Part of $\mathrm{PhD}$ degree thesis, Postgraduate Program in Thoracic and Cardiovascular Surgery. Tutor: Prof. Roberto Costa. 